\title{
Analysing the stromal microenvironment in vestibular schwannomas - key to their growth?
}

\section{Paramita Baruah, Jennifer Marshall, Peter Monksfield, Richard Irving, Christopher Buckley \\ Department of ENT, University Hospital Birmingham and Institute of Ageing and Inflammation, Birmingham University, UK}

\section{Background}

Vestibular schwannomas (VS) remain a challenge despite improvements in surgery and radiotherapy. Strategies to target tumor microenvironment to shrink VS before surgery or radiotherapy could vastly improve the outcomes of management.

Fibroblasts and macrophages form an important part of the stroma in VS and could support tumour growth

Very little is known about fibroblasts and macrophages in the VS microenvironment

\section{Aim}

To establish fibroblast cultures from VS and analyse their phenotype and function

To analyse fibroblasts and macrophages in VS tumour tissue

\section{Methodology}

Patients undergoing VS surgery were recruited into the study. Tumour tissue was used to establish fibroblast cultures.

Immunofluorescence staining for fibroblast markers was performed on frozen tumour tissue

Immunofluorescence for macrophage markers was performed on forzen tumour tissue

\section{Results - 1}

Number of patients in study : 17 (13 female and 4 male)

Age range: $31-76$ years (mean age $=54$ years)

Fibroblasts cultures were successfully established from 10 of the 17 patients recruited (Figure 1)

A strong expression of fibroblast markers CD90 and podoplanin was present in all VS samples on immunofluorescence (Figure 2 and 3 )

The stem cell marker CD34 was expressed in $50 \%$ of the samples (Figure 2 and 3 ).

Macrophage marker CD163 was present in $60 \%$ of VS samples (Figure 4).

\section{Figure - 1}
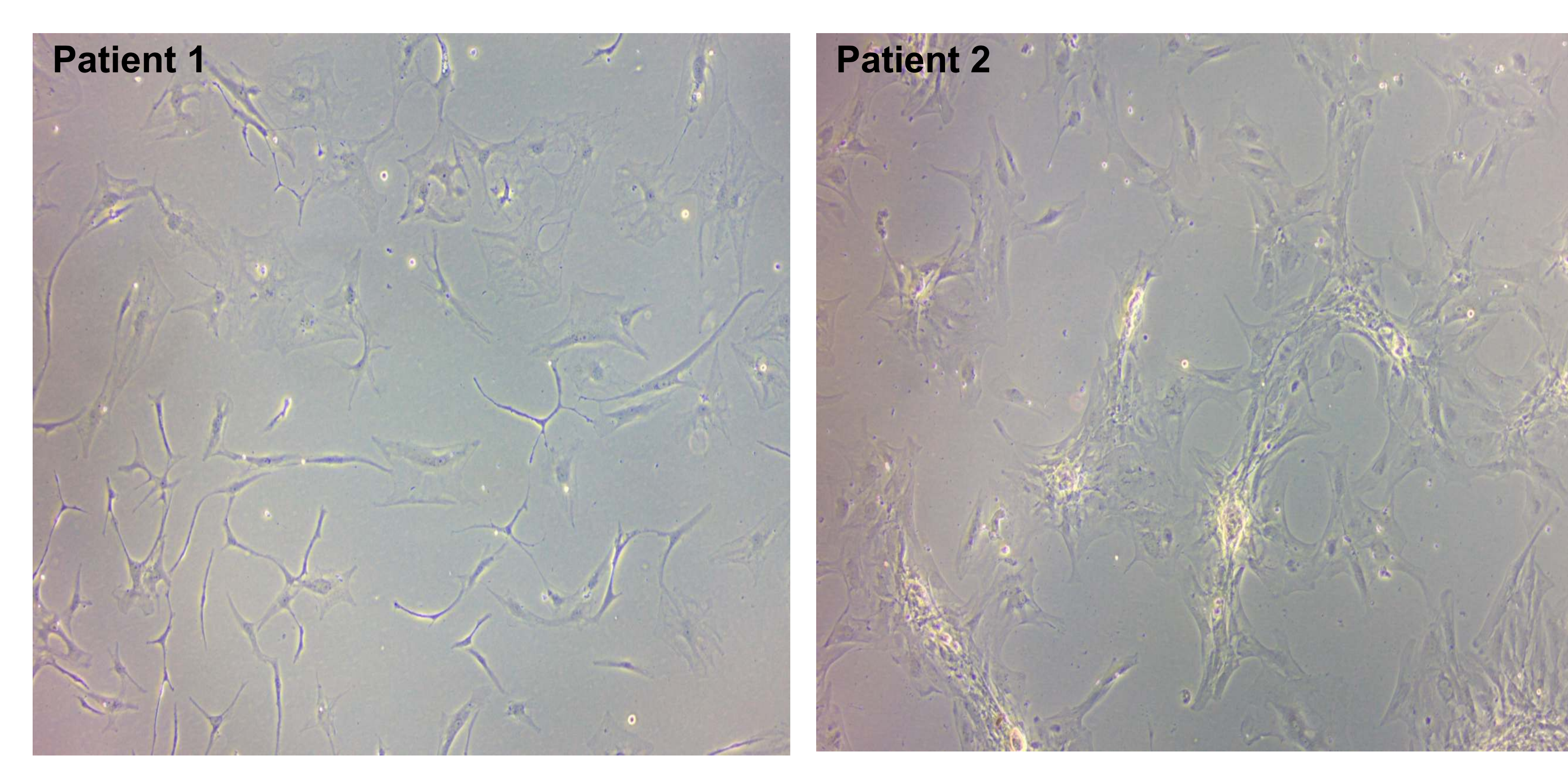

Figure - 2

Isotype control

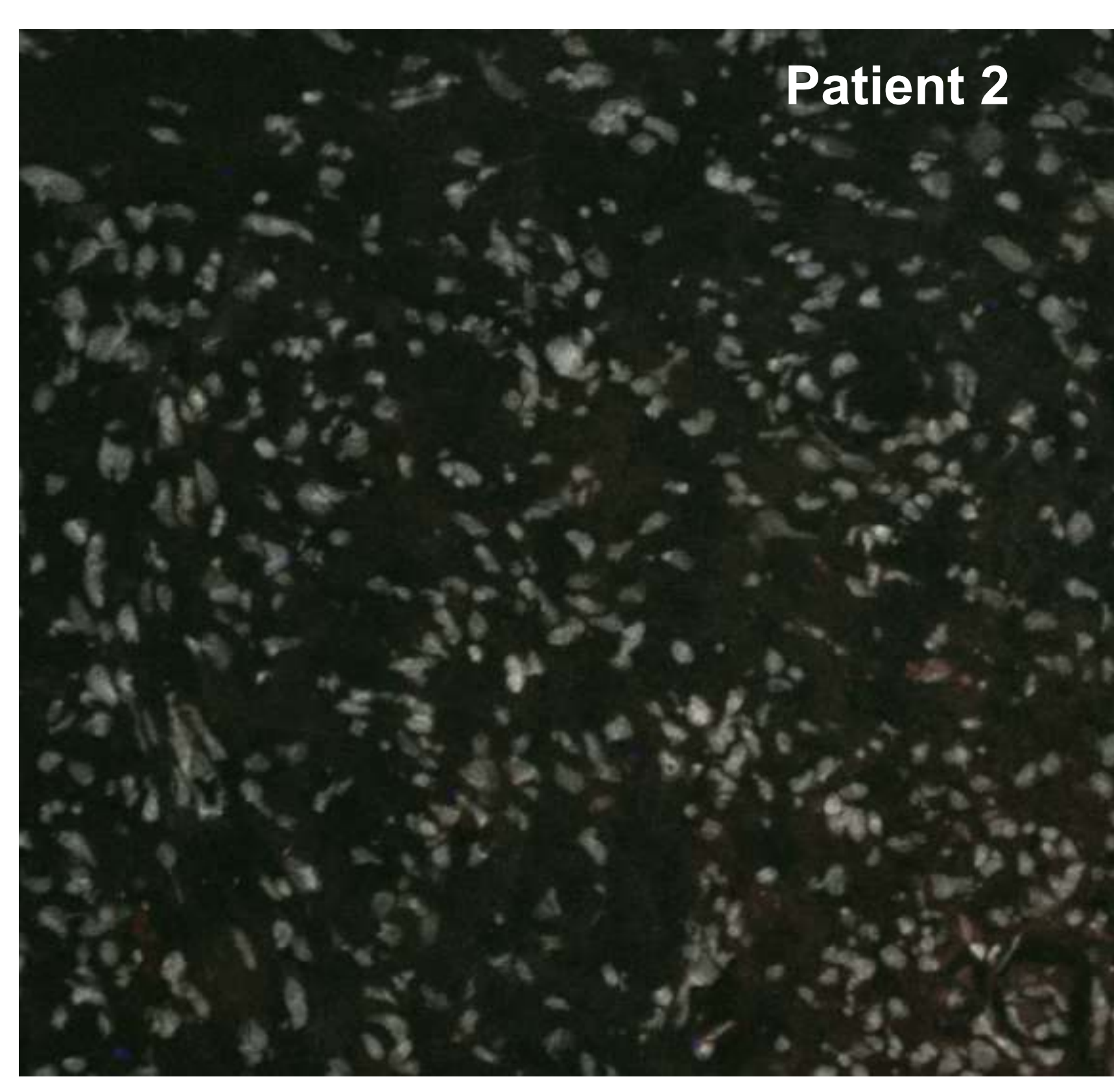

Figure - 3

CD90 (green) Podoplanin (blue) CD34 (red)
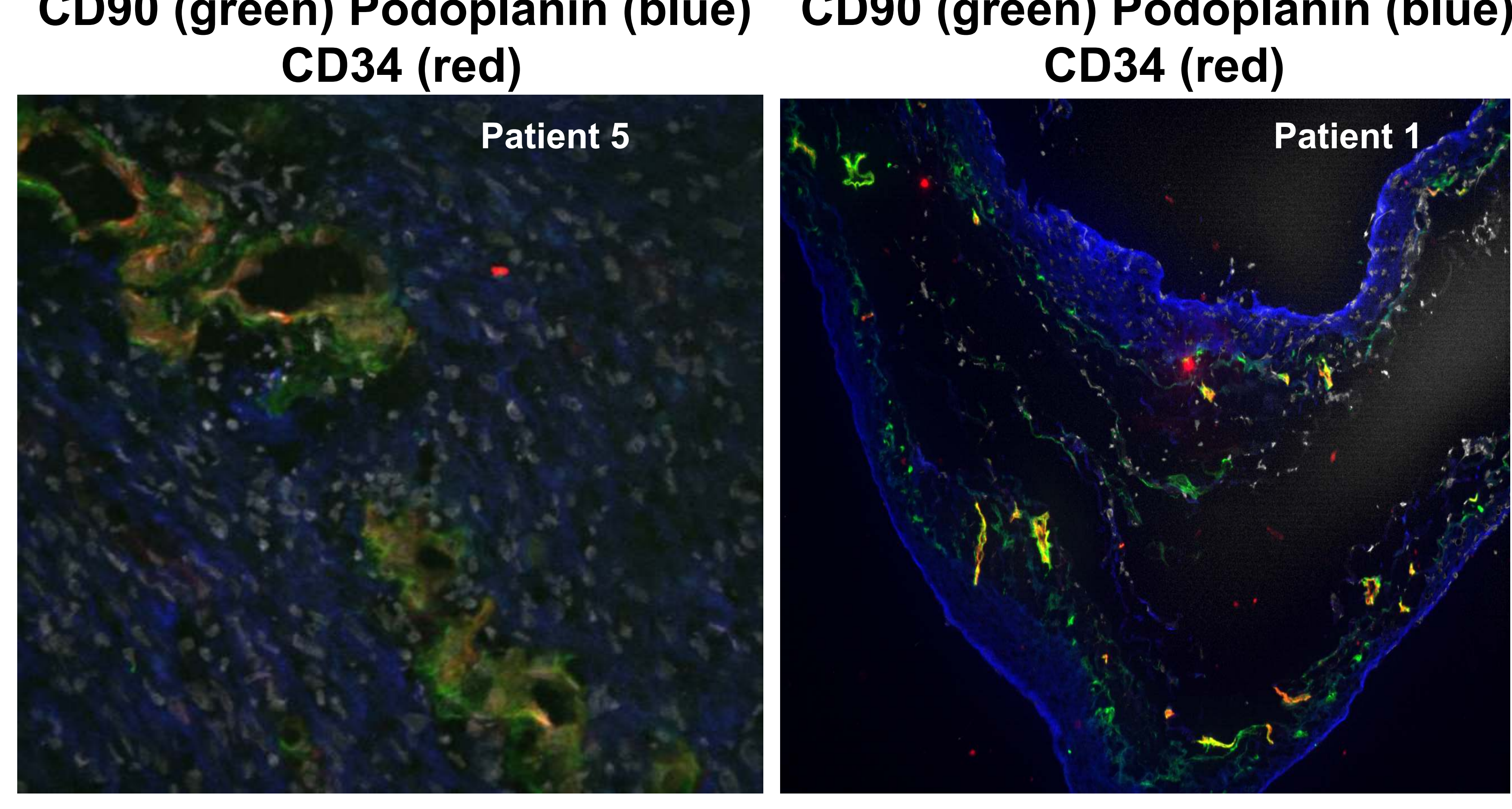

Figure - 4

CD163 (red)

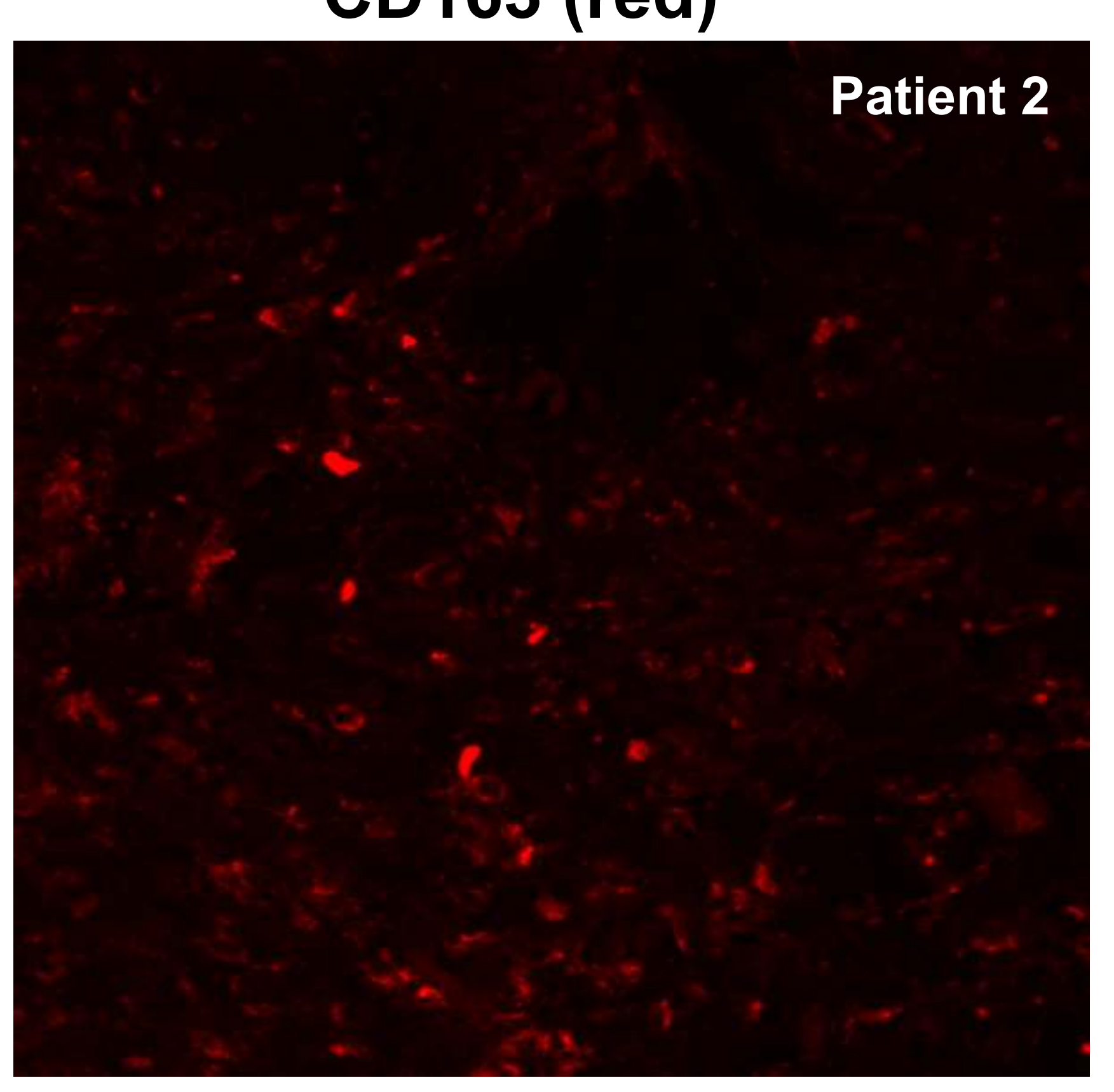

CD90 (green) Podoplanin (blue) CD34 (red)

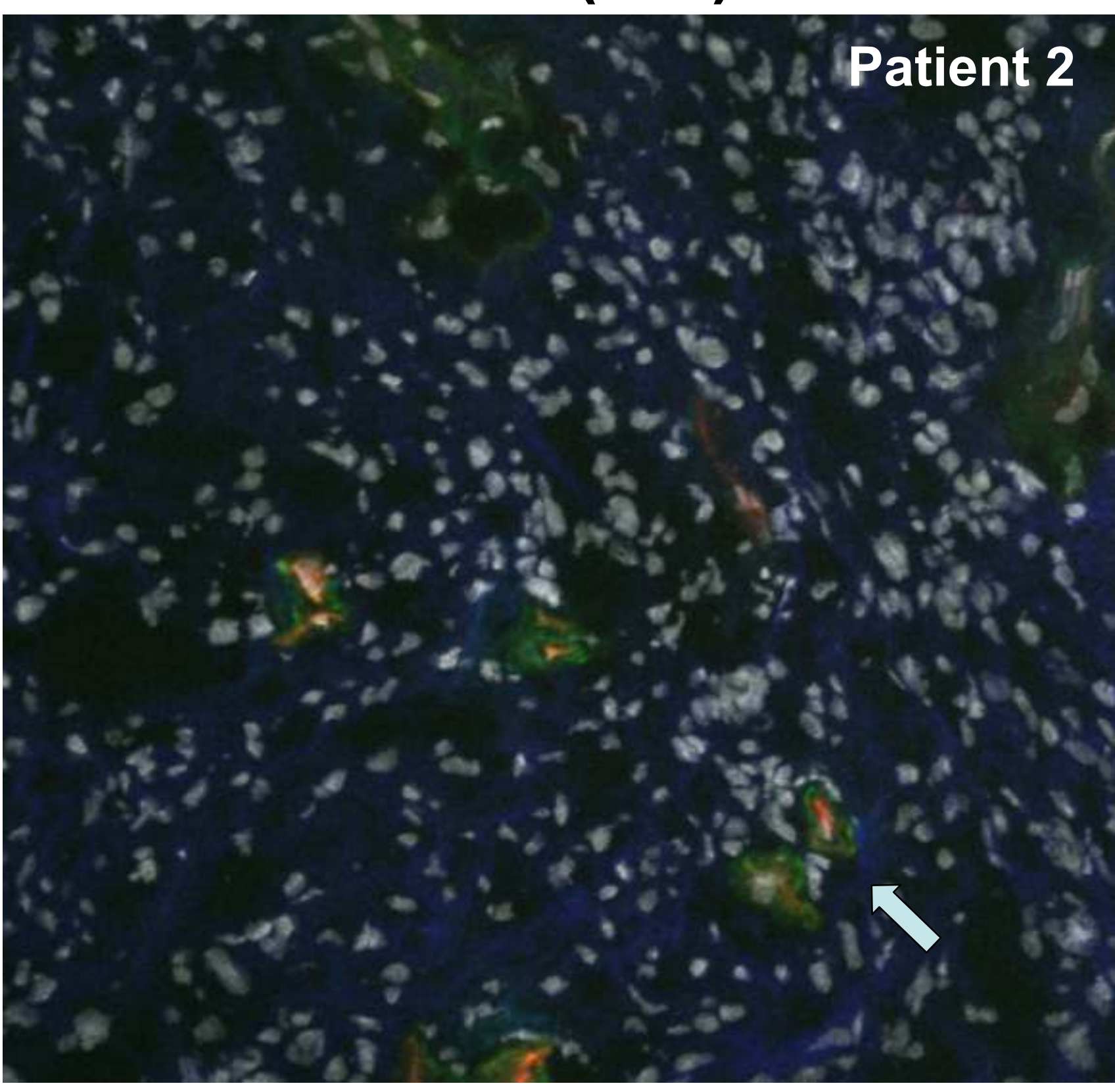

